\title{
The Role of Male Teachers as Perceived by Daycare Center Directors
}

\author{
Kwang-seop Kim ${ }^{1}$, Jeong-Moo Hurh ${ }^{2}$ \\ ${ }^{1}$ Doctorate Course, Department of Education, Jungwon University, Korea, sub4587@nate.com \\ ${ }^{2}$ Professor, Dept.of Early Childhood Education, Korea National Univ. of Transportation, \\ Korea,jmhuh@ut.ac.kr \\ Corresponding Author: Jeong-Moo Hurh
}

\begin{abstract}
The purpose of this study is to investigate the role of male childcare teachers in daycare centers as perceived by the directors. Forty-five directors with experience in hiring male childcare teachers were selected as the subjects. The survey was conducted using a questionnaire on the job and role of male childcare teachers in daycare centers. A frequency analysis was performed on the questionnaires collected after surveying for two months from September 2020. Based on the result of the study, it was found that the reason why there are few male teachers in daycare centers is because of the perception that it is a female field, and there is concern about the high proportion of female teachers. The expected roles of male daycare teachers were found to be physical and outdoor activities and guidance on the life of young male children. The following conclusions were drawn. First, the reason why there are few male childcare teachers in daycare centers is due to the recognition that child care is for females and with low socioeconomic status. Second, despite the low awareness of male childcare teachers' vocational stability and social status, daycare center directors recognize that male childcare teachers' sense of mission and integrity for early childhood education, and inclusion of young children are important qualities. Third, the perceptions of the directors on the role of male childcare teachers are positive. They accepted the male childcare teachers because male staff members can provide help in physical and outdoor activities as well as life guidance for young male children.
\end{abstract}

Keyword: Male Childcare Teacher, Preoccupation of Female Childcare Teacher, Daycare Center, Daycare Center Director

\section{Introduction}

In daycare centers, young children satisfy their social needs through interactions not only with fellow children, but also with childcare teachers. The relationship with teachers in daycare centers is very important because it provides the basis for experiencing and continuing development of various human relationships from the moment they get out of the first social environment, the interaction with their parents at home, and when they are sent to a daycare center.

In the process of human development, the development of gender roles is formed through human relationships. Likewise, gender role stereotypes acquire knowledge comparable to adulthood when they reach the age of six, and the concept of gender roles acquired through early childhood education institutions in young child continues to influence human relationships with people around them until adulthood.

Aside from the importance of acquiring the concept of gender roles through relationships with

Received: February 17, 2021; 1st Review Result: April 01, 2021; 2nd Review Result: May 21, 2021 Accepted: June 30, 2021 
childcare teachers in daycare center, it is also necessary to understand gender role socialization from different perspectives, such as male and female childcare teachers. In the meantime, since the human composition of male childcare teachers accounted for a relatively small distribution in the young children education field, it was true that studies on the influence of male childcare teachers on the socialization of the gender role of young children were poor. Therefore, by expanding the interest in socialization of gender roles that female childcare teachers have so far, it is important to find out the importance of socializing gender roles in young children that male childcare teachers have, and what gender role characteristics are expected and emphasized by daycare centers for male childcare teachers.

The acquisition of the concept of gender roles in young children is achieved primarily through relationships with parents at home and secondarily at daycare centers with childcare teachers. The gender role socialization[1] is achieved from different viewpoints of male childcare teachers and female childcare teachers. However, in the childcare field, since the human composition of male childcare teachers accounted for a relatively small distribution, interest in the influence of male childcare teachers on the socialization of the gender role of young children was insufficient. The phenomenon of feminization of the teaching profession is accelerating in education and childcare sites, and the degree of acceleration in elementary schools as well as daycare centers and kindergartens adds to its seriousness. There are lesser male teachers than female teachers because the early childhood education and childcare is based on the social perception that the jobs of early childhood teachers and childcare teachers are feminine[2][3]. Therefore, it is important to find out the importance of socializing gender roles in young children that male childcare teachers have, their expected roles, and their characteristics in daycare centers.

Male childcare teachers have the advantage of being able to interact more physically because they are more active in play activities among childcare activities in daycare centers[4]. Nevertheless, it cannot be denied that the cases of child sexual abuse reported in the media have abnormally reinforced the image of male childcare teachers. As a result, it cannot be overlooked that it resulted in the reduction of the range of childcare activities of male childcare teachers[5].

Contrary to these social prejudices and concerns, the positive effects of male childcare teachers, that is, the role and care of male childcare teachers, are positive for the development of young children, and are expected to be an important socialization model for boys[6][7]. It is expected that male childcare teachers will be able to provide experiences and learning different from those of female childcare teachers. This is because they can understand boys' viewpoints and experiences as a man, and male childcare teachers consider it to be the most important responsibility they have to carry out[8] that they should provide an ideal male role model for girls as well as boys.

Therefore, the purpose of this study is to investigate on the perception of the preoccupation of female teachers in daycare centers and the perceptions of the directors of daycare centers on the role of male teachers. It is aimed to provide basic data to seek ways to re-establish the roles of male teachers in daycare centers by identifying the perception of the preoccupation of female teachers in daycare centers and the expected roles of male childcare teachers.

Two research questions were set to achieve the research objectives.

First, what is the director's perception of the preoccupation of female childcare teachers in daycare centers?

Second, what are the expectations of the director on the role of male childcare teachers in daycare centers?

\section{Related Prior Research}

The gender distribution of male and female teachers is an important factor in the composition of the human environment of daycare centers. The reason why the number of male daycare teachers has been 
so small is the fear of the possibility that physical contact with infants can develop into sexual problems. For this reason, it can be inferred that the interactions with young children were reduced, and educational activities could not be reduced as a result of being overly cautious by controlling their behavior so as not to be misunderstood.

In the educational field, the phenomenon of feminization of the teaching profession is accelerating. The degree of acceleration in early childhood education and primary education adds to its seriousness. In the field of child care, the reason why male childcare teachers are less than female childcare teachers is based on the social perception that the job of childcare teachers is a feminine career.

The reason why the discussion on the feminization of the teaching profession in the child care field was sluggish in the field of child care is that caring for infants and young children is a woman's job, and the perception of the general society is not very concerned about the concentration of female teachers.

It is also an important reason that the welfare level and social status of childcare teachers are inferior to that of elementary and secondary teachers. Traditionally, the perception that men were the supporters of family livelihoods predominated, so it can be said that men hesitated and avoided entering the child care field, which had been accompanied by low pay and social status. Because the environment of daycare centers working with teacher training institutions is dominated by a female-centered culture, male childcare teachers are having difficulties and avoid them. Most of the members of the child care field are women, and they are creating the culture of the child care field in a way that they are comfortable and familiar with. Few men have a hard time understanding and adapting to the culture of women who differ from them.

The preoccupation of female teachers in daycare centers has limitations in that it can instill false stereotypes about gender roles in young children, and there is no male role model for boys to model. When all the faculty and staff of daycare centers are made up of women, children think that child care teachers are only women's occupations, so they are aware of the wrong gender role, and furthermore, education and parenting, the main functions of childcare, In the aspect of learning from an early age the thought that only women are in charge of women, the side effects of women's bias can occur. Because many female teachers treat young children's female behavior positively and male behavior negatively, they prefer children who follow behavioral norms such as politeness, cleanliness, obedience, and cleanliness. It is expressing concern that it may lead to negative consequences for unhealthy male children.

As concerns have been raised that the feminization of the teaching profession, which has emerged since the 1970s, can make infants feminine, the main concern of concern has been pointed out as a problem, in particular, the feminization of boys. Because male teachers satisfy the desire for paternity and promote the social development of young children, they can help both male and female children adapt and enrich their educational experience. In particular, the necessity can be justified because the male infant teacher is expected to be able to compensate for the absence of a father in the home as a male role model for a boy in a family without a father.

The dominant view is that men and women should equally share the responsibilities of child care, since a genuine change in the orthodox gender role cannot occur unless men and women participate equally in parenting. In that case, it is believed that male childcare teachers can break young children from the traditional gender role stereotype and ultimately contribute to the realization of gender equality.

The necessity and importance of male childcare teachers in daycare centers can be summarized in four ways.

First, from the perspective of gender equality education, male childcare teachers are needed from the anti-bias perspective of child care, which places importance on diversity and wide experience. This is because male childcare teachers can help boys and learn that many members of society are helping and living in different ways, and they can help them recognize and agree on this diversity.

Second, a male childcare teacher is needed because it can make the father, the male parent of young 
children feel more responsible, as a complementary perspective on fatherlessness. When a male childcare teacher of the same sex as the father experiences parenting or educational activities, the father himself becomes aware of more responsibility for the children's parenting and educational activities.

Third, it is positive that the number of male childcare teachers increases in the professional development of the teaching profession, so male childcare teachers are needed. In a male-centered society, occupations in which men are the mainstream have good professional status and treatment, whereas when women are the center of the profession, their professional expertise is low and the pay is poor.

Fourth, when an infant teacher is composed of only female teachers, young children recognize the wrong gender role as an occupation for young children only women, and furthermore, childcare and parenting activities can be considered as an area dedicated only to women, so a male childcare teacher is necessary.

Recently, a new awareness of the necessity and importance of male childcare teachers has emerged, and a new awareness of the role of male childcare teachers has increased, thus, research on male childcare teachers is expanding.

According to the results of a study by Daewook Kim[9] who studied the difficulty of adaptation and the search for job turnover experienced by first-time male kindergarten teachers, it turns out that they are experiencing different worries from female teachers, such as being unable to adapt to work environment, having difficulty on establishing relationships, religious conflicts, and personal dispositions for the full-time teachers.

According to the research results of Sewoong Kim[6] who studied the appearance of male teachers reflected by female teachers in early childhood education institutions, they were poor in reading the deep innermost of children in interaction, expressing more straight forward than female teachers, and relatively compared to other areas. It was recognized as showing a tendency to neglect life guidance. As a childcare teacher, a male teacher had difficulties in dealing with young children and bowel movements, which was the greatest distrust of parents due to social awareness, and even female teachers working together were found to be distrustful of male teachers in this regard. On the other hand, on the positive side, male teachers have a positive influence on internalizing the gender role of young children in early childhood education institutions with a large number of women, and address the needs of young children through active and diverse methods unique to physical activity and play. Male teachers are good at repairing facilities, handling electronic devices well, and taking the initiative in events. These strengths have been shown to help them cover their shortcomings and recognize them as necessary.

In-depth interviews with six male childcare teachers, interviews with neighbors, participatory observation, and research participants such as journals to reveal the experiences and essential meanings of male childcare teachers who live in small numbers among many female teachers experience in the process of becoming a teacher. There is a study by Kwon So-young[10] that collected personal documents of the children and analyzed them with a qualitative research method. Participants in the study recognized a clear difference with female teachers from the days of preparatory teachers, and these differences were becoming more concrete as they faced difficulties living as early childhood teachers. Contrary to the preconceived notion that men would not be suitable for caring, the study participants were fulfilling their duties as teachers by pursuing the essential meaning of education through genuine encounters with young children. It turned out that male childcare teachers are living in a multi-layered conflict in which male teachers, who are socially and physically strong, have to live as weak rather than in a group of female teachers.

According to the research results of Seung-Hyun Cho[11], which investigated the change of parents' perceptions of male teachers in early childhood education institutions, the male and female childcare teachers should have the same ratio after the male teacher was in charge of the classroom teacher. Positively, the perception has changed. However, with regard to the recognition of the qualifications and 
roles of male early childhood teachers, natural education on gender equality was suggested as the most important role, but it was investigated that there were many areas of concern about the physical contact of male early childhood teachers.

According to the research results of Eun-Hye Roh[5], who explored the difficulties of male childcare teachers using the $\mathrm{Q}$ methodology, male childcare teachers were found to experience a lot of difficulties.

According to the research result of Lee Kun-koo[4], which compared the perceptions of school staff and parents about male childcare teachers, the reason why there were few male childcare teachers in daycare centers was a strong perception of women's field and negative social status, stability, and prospects. It points out that the perception was strong. As for the necessity of male childcare teachers, it was found that the faculty and staff were stronger than the parents, and they recognized that male childcare teachers played a more important role in the education and care of young children and event management.

According to the research results of Sang-yeol Lee[1] on the effect of the gender role stereotype of female childcare teachers on the role perception of male childcare teachers, the higher the gender role stereotype of female childcare teachers, the lower they perceived the role of male childcare teachers.

In summary, it can be seen that the research on male childcare teachers is expanding in the field of early childhood education and childcare, and the awareness of the necessity and value of male childcare teachers is spreading. The importance and value of socializing the gender role of male childcare teachers has been verified through many empirical research results despite the number of female teachers in the childcare field, where the proportion of female teachers accounts for the majority. Specific research and discussions to support will have to be conducted continuously.

\section{Method}

In this study, a total of 45 of daycare centers located in Chungcheongnam-do and Chungcheongbukdo with experience of hiring male childcare teachers were selected as the subject. Since the number of daycare centers employing male daycare teachers is limited, all directors of the relevant regions were selected for the survey. Of the 58 directors, 45 cooperated with the survey, all of them were women, and the ages were 25 in their 40 s and 20 in their 50s. The survey was conducted using a questionnaire on the job and role of male childcare teachers at daycare centers. The questionnaire was composed of nine questions, and as a result of calculating the Cronbach's a coefficient, which is an intra-item fit, it was found to be .92, securing a high level of reliability. From September 1 to October 15, 2020, a survey was conducted to the directors of the study through e-mail, and the collected questionnaire was analyzed by applying the SPSS 23.0 statistical program. As the data analysis method, frequency analysis was applied.

Prior to conducting the research, approval was obtained from IRB of the researcher's affiliated institution. In order to secure the ethics of the research, the purpose, method, and process of the research were explained in detail to the research participants during the research process, and the research was conducted with the participants who voluntarily expressed their intention to participate in the research. It was fully explained that even if you agreed to participate in the study, can withdraw if do not want to participate in the process. When analyzing research data and stating the results, all personal information or information that can identify an individual was encoded and recorded. Research data was promised to the participants to be destroyed after storage for 3 years. The researcher tried not to interfere with the subjectivity and preconceived notions in the process of research through describing and reflecting on the research topic before conducting the study. 


\section{Results}

\subsection{Recognition of the Preoccupation of Female Teachers in Daycare Centers}

[Table 1] shows the results of a survey on how the directors perceive the reason why there are few male teachers in daycare centers.

[Table 1] The Reason Why the Number of Male Teachers is Low

\begin{tabular}{cc}
\hline Answer & $\mathrm{N}(\%)$ \\
\hline Low social status & $7(15.2)$ \\
\hline Recognition as a women's field & $28(62.6)$ \\
\hline The idea that it doesn't fit the man's aptitude & $10(22.2)$ \\
\hline Total & $45(100.0)$ \\
\hline
\end{tabular}

According to [Table 1], as for the reason that there are few male teachers in daycare centers, the directors have answered 'Recognition as a women's field' the most with 29 (62.6\%) responses, followed by 'The idea that it doesn't fit the men's aptitudes' with $10(22.2 \%)$ responses, and by 'Low social status' with $7(15.2 \%)$ responses.

[Table 2] shows how the directors perceive that the majority of childcare teachers are women.

[Table 2] Recognition that the Majority of Childcare Teachers are Women

\begin{tabular}{cc}
\hline Answer & $\mathrm{N}(\%)$ \\
\hline It is a desirable phenomenon & $20(44.4)$ \\
\hline This is a worrisome phenomenon & $25(55.6)$ \\
\hline Total & $45(100.0)$ \\
\hline
\end{tabular}

According to [Table 2], the directors' perception on the recognition of majority of childcare teachers are women indicated that 'this is a worrisome phenomenon' with 25 (55.6\%) responses and 'it is a desirable phenomenon' with $20(44.4 \%)$ responses.

[Table 3] shows the results of the survey on the reasons for the directors think that it is desirable to have a high proportion of female teachers.

[Table 3] Reasons for Approving the High Proportion of Female Teachers

\begin{tabular}{cc}
\hline Answer & $\mathrm{N}(\%)$ \\
\hline Because women have a natural parenting instinct & $7(35.0)$ \\
\hline Young children need delicate and thorough care & $10(50.0)$ \\
\hline Because female teachers are better than male teachers & $3(15.0)$ \\
\hline Total & $20(100.0)$ \\
\hline
\end{tabular}

According to [Table 3], the directors' perceptions on the reason why the ratio of female teachers is desirable indicated that 'Young children need delicate and thorough care' with 10 (50.0\%) responses, followed by 'Because women have a natural parenting instinct' with 7 (35.0\%) responses, and 'Because female teachers are better than male teachers' with $3(15.0 \%)$ responses.

On the contrary, in the case of directors who believe that the high proportion of female teachers is a phenomenon to be concerned about, the results of a survey on the reason are as shown in [Table 4]. 
[Table 4] Reasons for Worrying about the High Proportion of Femal Teachers

\begin{tabular}{cc}
\hline Answer & $\mathrm{N}(\%)$ \\
\hline The feminization of boys & $6(24.0)$ \\
\hline Because physical or outdoor activities are hindered & $3(12.0)$ \\
\hline It is necessary for young children to experience male and female teachers evenly & $16(64.0)$ \\
\hline Total & $25(100.0)$ \\
\hline
\end{tabular}

According to [Table 4], the directors' perception on the reasons why they think that the high proportion of female teachers is a phenomenon to be concerned about indicated that 'It is necessary for young children to experience male and female teachers evenly' with $16(64.0 \%)$ responses, followed by 'The feminization of boys' with $6(24.0 \%)$ responses, and 'Because physical or outdoor activities are hindered' with $3(12.0 \%)$ responses.

\subsection{Recognition of Male Childcare Teachers as a Profession}

[Table 5] shows how the directors perceived the social status of male childcare teachers.

[Table 5] Awareness of Male Childcare Teachers' Social Status

\begin{tabular}{cc}
\hline Answer & $\mathrm{N}(\%)$ \\
\hline Will be high & $-(0.0)$ \\
\hline Will be normal & $22(48.9)$ \\
\hline Will be low & $23(51.1)$ \\
\hline Total & $45(100.0)$ \\
\hline
\end{tabular}

According to [Table 5], as with the social status of male childcare teachers, the directors showed a near distribution of responses responding to 'will be low' (23 or 51.1\%) and 'will be normal' (22 or $48.9 \%)$.

[Table 6] shows the results of the survey on how the directors perceive whether male childcare teachers work for a long time.

[Table 6] Thoughts on Whether Male Childcare Teachers are Working for a LongTtime

\begin{tabular}{cc}
\hline Answer & $\mathrm{N}(\%)$ \\
\hline Will work longer than the female teachers & $2(4.4)$ \\
\hline Will be moving faster than female teachers & $15(33.3)$ \\
\hline There are individual differences, but no special differences & $28(62.3)$ \\
\hline Total & $45(100.0)$ \\
\hline
\end{tabular}

According to [Table 6], the thoughts on whether male childcare teachers will work for a long time indicated that 'There are individual difference, but no special differences' with 29 (62.3\%) responses, followed by 'Will be moving faster than female teacher' with 15 (33.3\%) responses, and 'Will work longer than a female teacher' with $2(4.4 \%)$ responses.

[Table 7] shows how the directors perceive what is the most important qualities of male childcare teachers. 
[Table 7] The Most Important Qualities of Male Childcare Teachers

\begin{tabular}{cc}
\hline Answer & $\mathrm{N}(\%)$ \\
\hline Affection and inclusion for young children & $16(35.5)$ \\
\hline A modest attitude toward young children & $4(8.9)$ \\
\hline Commitment, fairness, and sincerity for child care & $22(48.9)$ \\
\hline Philosophy and expertise in child care & $3(6.7)$ \\
\hline Total & $45(100.0)$ \\
\hline
\end{tabular}

According to [Table 7], among the most important qualities of male childcare teachers, the directors perceived 'Commitment, fairness, and sincerity for childcare' with 22 (48.9\%) responses, followed by 'Affection and inclusion for young children' with 16 (35.5\%) responses, 'A modest attitude toward young children' with 4 (8.9\%) responses, and 'Philosophy and expertise on child care' with 3 (6.7\%) responses.

\subsection{Recognition of the Role of Male Childcare Teachers}

[Table 8] shows the results of the survey on how the directors perceive the part that is helpful when working with a male childcare teacher.

[Table 8] What is Helpful When Working with a Male Teacher

\begin{tabular}{cc}
\hline Answer & $\mathrm{N}(\%)$ \\
\hline Natural education on gender equality & $18(40.0)$ \\
\hline Physical and outdoor activities & $17(37.8)$ \\
\hline Life guidance of young male children & $7(15.6)$ \\
\hline Regardless of gender, teachers' abilities and qualities come first & $3(6.6)$ \\
\hline Total & $45(100.0)$ \\
\hline
\end{tabular}

According to [Table 8], the most helpful part of working with a male childcare teacher was 'Natural education on gender equality', with 18 (40.0\%) responses, followed by 'Physical and outdoor activities' with $17(37.9 \%)$ responses, 'Life guidance of male young children' with 7 (15.6\%) responses, and 'Regardless of gender, teacher's abilities and qualities come first' with $3(6.6 \%)$ responses.

[Table 9] shows the results of a survey on how the directors perceived the role that male childcare teachers would perform.

[Table 9] Expected Roles for Male Childcare Teachers

\begin{tabular}{cc}
\hline Answer & $\mathrm{N}(\%)$ \\
\hline Working that requires physical strength & $22(48.9)$ \\
\hline Computer and machine management & $2(4.4)$ \\
\hline Life guidance of young male children & $6(13.4)$ \\
\hline Promotion of young children's physical development & $15(33.3)$ \\
\hline Total & $45(100.0)$ \\
\hline
\end{tabular}

According to [Table 9], the directors perceived the role that male childcare teachers are particularly expected to do included 'Work that requires physical strength' with 22 (48.9\%) responses, followed by 
'Promotion of young children's physical development' with 15 (33.3\%) reponses, 'Life guidance of male young children' with 6 (13.4\%) reponses, and 'Computer and Machine Management' with 2 (4.4\%) responses.

\section{Conclusion and Suggestions}

The expectations and perceptions of the heads of daycare centers on the roles and value of male childcare teachers are increasing. Thus, the need for research in this field is also increasing. Based on the results of the investigation, the following conclusions were drawn.

There are few male childcare teachers in daycare centers because of the recognition that young children care is a female field and reflects a low socioeconomic status. The proportion of female childcare teachers is high and a concern among directors in terms of gender role education, difficulties in physical or outdoor activities, and feminization of boys.

Nevertheless, many directors are not very concerned about the preoccupation of female teachers in day care centers because young children require delicate and detailed education and women have innate parenting instincts.

Despite the low awareness of male childcare teachers' occupational stability and social status, daycare center directors recognize that male childcare teachers' sense of mission and integrity for childcare, and inclusion of young children are important qualities.

The perception of daycare center directors about the role of male childcare teachers is positive. They are optimistic about accepting male childcare teachers because they can receive help in natural education on gender equality, physical and outdoor activities, and life guidance for young male children. Mainly, work that requires physical strength in daycare centers, promotion of physical development of young children, life guidance of male young children, and computer and machine management are expected as important roles of male childcare teachers.

Based on the research results and conclusions, the following suggestions are made for further research on increasing awareness of the necessity, value, and role of male childcare teachers.

First, this study was limited to quantitative studies that analyzed the perceptions of daycare center directors. Therefore, there is a limit in the aspect of a qualitative study to find out why the directors' personal background characteristics and work experience with male childcare teachers are related to the recognition of the necessity and role of male childcare teachers. Therefore, in order to re-establish a new perception and role of male teachers, which are gradually increasing in daycare centers, a complex study is needed to conduct qualitative research such as in-depth interviews, content analysis, and participatory observation. When various research methods that combine unstructured methods such as in-depth interviews and observational studies are applied, a broad understanding of the role and job performance characteristics of male childcare teachers is possible. In addition to the general perception of the role of male teachers in daycare centers, research is required to verify the educational influence of male childcare teachers through experimental studies conducted in classroom situations and to compare them with female childcare teachers.

In the results of this study, it was found that the perceptions of the directors of daycare centers about the role of male childcare teachers were generally positive, but their perceptions could also be seen to reflect social stereotypes. Therefore, while research on male childcare teachers in daycare centers is more expanded, anti-bias education and gender equality education that eliminate prejudice against male childcare teachers and the stereotype that young children care is a female field should be initated. To this end, social efforts to enhance the professional status of male childcare teachers and the role of the mass media for improving awareness of male childcare teachers and delivering accurate images are important to the general public. As suggested in this study result, the fact that the directors of daycare centers who had experience of hiring and working with male childcare teachers were more positive 
about the role and job performance of male childcare teachers.

Since this study is a study targeting directors who are hiring male childcare teachers among daycare centers located in Chungcheongnam-do and Chungcheongbuk-do, there is a limit to the generalization of the results. For follow-up studies, a nationwide study is requested. In addition, a study is needed to compare the perceptions of male daycare teachers' roles by surveying male and female daycare teachers. In this study, the research was conducted focusing on the questionnaire survey, but in-depth interview studies and case studies are required for male childcare teachers and the directors who employ them.

\section{References}

[1] S. Y. Lee, Effects of Gender Role Stereotypes of Female Childcare Teacher on The Role Recognition of Male Childcare Teachers, Daegin University, Master's thesis, (2020)

[2] S. Y. Park, Female Teachers' and Staff Members' Perceptions of Male Teachers in Early Childhood Education, ChungAng University, Master's thesis, (2019)

[3] B. H. Moon, H. K. Hong, The Comparison of Awareness on Male Teacher between In-service Teachers and Pre-service Teachers, Early Childhood Education Research \& Review, (2011), Vol.15, No.2, pp.91-107, UCI : G704000814.2011.15.2.020

[4] G. G. Lee, A Comparative Study of Teachers and Parents' Perception of Male Infant Teachers, Kyonggi University, Master's thesis, (2018)

[5] E. H. Rho, Exploring difficulties of male child care teachers using Q methodology, Kyung Hee University, Master's thesis, (2017)

[6] S. U. Kim, Image of male teachers from the perspective of female teachers in early childhood education institution, Paichai University, Master's thesis, (2015)

[7] S. H. Baek, J. H. Noh, Qualitative research on "becoming teacher" of male early childhood teachers, The Journal of Eco Early Childhood Education \& Care, (2013), Vo.12, No.1, pp.49-73, UCI : G704-002046.2013.12.1.004

[8] S. Y. Park, The stories of male early childhood teachers who have worked for more than 5 years, Paichai University, Master's thesis, (2020)

[9] D. W. Kim, A Study on Adaptation and Turnover of Novice Male Kindergarten Teachers, Early Childhood Education Research \& Review, (2013), Vol.17, No.1, pp.339-365, UCI : G704-000814.2013.17.1.014

[10] S. Y. Kwon, Being a minority Male Teacher in Early Childhood Education, Sookmyung Women's University, Master's thesis, (2014)

[11] S. H. Cho, A study on changes in parents' recognitions of male early childhood teachers in educational institutions for young children, Kongju National University, Master's thesis, (2015) 